\title{
Oxygen Vacancies as Active Sites for Water Dissociation on Rutile $\mathrm{TiO}_{2}(110)$
}

\author{
R. Schaub, ${ }^{1}$ P. Thostrup, ${ }^{1}$ N. Lopez, ${ }^{2}$ E. Lægsgaard,${ }^{1}$ I. Stensgaard, ${ }^{1}$ J. K. Nørskov, ${ }^{2}$ and F. Besenbacher ${ }^{1, *}$ \\ ${ }^{1}$ Institute of Physics and Astronomy and CAMP, University of Aarhus, DK-8000 Aarhus, Denmark \\ ${ }^{2}$ Department of Physics and CAMP, Technical University of Denmark, DK-2800 Lyngby, Denmark
}

(Received 30 July 2001; published 6 December 2001)

\begin{abstract}
Through an interplay between scanning tunneling microscopy experiments and density functional theory calculations, we determine unambiguously the active surface site responsible for the dissociation of water molecules adsorbed on rutile $\mathrm{TiO}_{2}(110)$. Oxygen vacancies in the surface layer are shown to dissociate $\mathrm{H}_{2} \mathrm{O}$ through the transfer of one proton to a nearby oxygen atom, forming two hydroxyl groups for every vacancy. The amount of water dissociation is limited by the density of oxygen vacancies present on the clean surface exclusively. The dissociation process sets in as soon as molecular water is able to diffuse to the active site.
\end{abstract}

DOI: $10.1103 /$ PhysRevLett.87.266104

PACS numbers: 68.47.Gh, 68.37.Ef

While the description of metal and semiconductor surfaces has reached a high level of sophistication, many fundamental properties of the surfaces of metal oxides are still very poorly understood. Oxides are inherently much more complex, since most surface properties depend not only on the structure, but also on the local stoichiometry of the surface. The challenge is to understand how oxide surfaces react with gases, act as catalysts, photocatalysts, gas sensors, or act as supports for metal nanoparticles. The ambition is to be able to functionalize oxide surfaces by controlling composition and structure through defects or modifier atoms and molecules.

The rutile $\mathrm{TiO}_{2}(110)$ surface (see Fig. 1 and Ref. [1]) has become one of the key model systems for metal oxide surfaces [2]. A large number of experimental and theoretical studies have concentrated on this surface in the hope that it may act as a prototype system where consensus is first established. Water dissociation on $\mathrm{TiO}_{2}$ is of fundamental interest as an example of a simple surface chemical process with significant applications. In addition, $\mathrm{TiO}_{2}$ is the material of choice for photochemical hydrogen production from water $[3,4]$ and for biocompatible implants [5]. This further emphasizes the importance of understanding the water dissociation process in some detail. Earlier experimental studies report a minor degree of water dissociation on $\mathrm{TiO}_{2}(110)$ [6-8] while theoretical studies predict the dissociated state, or mixed dissociated-molecular states, to be most energetically favorable [9]. Thus, even for this simple process we do not have a clear picture of the dissociation energetics and the active site for dissociation.

In this Letter, we use a combined experimental and theoretical approach to show that, at low coverages, water dissociation takes place exclusively on defect sites associated with oxygen vacancies. By the use of scanning tunneling microscopy (STM) we demonstrate that a direct correlation between oxygen vacancies before water exposure and surface hydroxyl groups after exposure exists, and using density functional theory (DFT) we show water dissociation to be energetically feasible only at the defect sites.
An important part of the present study is the identification of the "type-A defects," a term introduced by Diebold and co-workers [10] to designate bright spots observed with STM on the $\mathrm{TiO}_{2}(110)$ surface. These defects are inherent to the surface, but their true chemical identity remains unresolved. Diebold et al. [10] interpret them as oxygen vacancies, while Suzuki et al. [11] associate them with hydroxyl groups. We show that there are actually two kinds of type-A defects, vacancies and hydroxyl groups, and high resolution STM images in combination with simulated STM images based on DFT calculations enable us to clearly distinguish the two. On this basis we conclusively identify the oxygen vacancy as the active site for water dissociation on titania. The onset of dissociation is limited by the mobility of the water molecules, which are found to adsorb on top fivefold coordinated titanium atoms.

The STM experiments were conducted in an ultrahigh vacuum (UHV) chamber, base pressure $<10^{-10}$ torr, equipped with standard sample preparation and analysis techniques. The STM used in these investigations is homebuilt [12] and capable of high-resolution scanning at

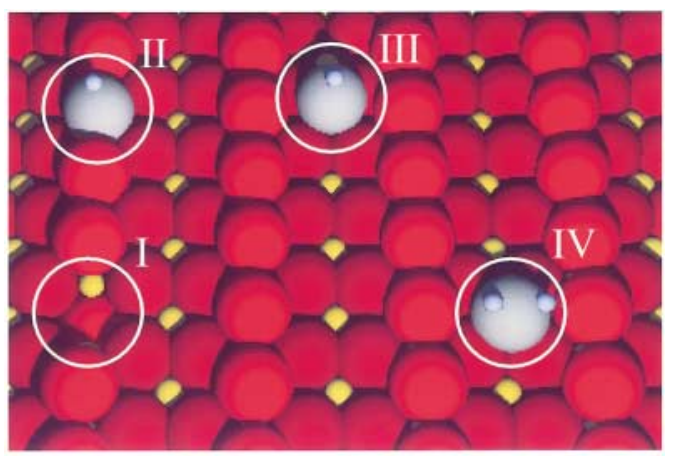

FIG. 1 (color). Ball model of the $\mathrm{TiO}_{2}(110)$ surface. The relevant included species on the surface are the following: (I) oxygen vacancy, (II) bridging hydroxyl group, (III) terminal hydroxyl group, and (IV) water molecule. Red atoms: O; yellow atoms: Ti; blue atoms: $\mathrm{H}$. 
100-400 K. The $\mathrm{TiO}_{2}$ samples (eSCeTe) were prepared by multiple cleaning cycles $\left(20 \mathrm{~min} 600 \mathrm{eV} \mathrm{Ar}^{+}\right.$sputtering, 40 min annealing at 1000-1100 K). Deionized water, cleaned via freeze-pump-thaw cycles, was introduced into the UHV chamber through a leak valve.

For theoretical modeling, we have performed DFT calculations applied to periodic slab models. Each slab contains three $\mathrm{O}-\mathrm{Ti}_{2} \mathrm{O}_{2}-\mathrm{O}$ planes and slabs are separated by $10 \AA$ of vacuum. Water is adsorbed on only one side, and the resulting dipole moment has been corrected for in the vacuum region. The ionic cores have been described by ultrasoft pseudopotentials [13] and the one-electron valence states are expanded in plane waves with kinetic energies up to $340 \mathrm{eV}$. A Monhkorst-Pack grid of $(8 \times 4 \times 1) \mathbf{k}$ points has been used for the smallest $(1 \times 1)$ surface unit cell and reduced appropriately for larger super cells. Electron densities have been self-consistently determined with the PW91 exchange-correlation functional [14] by iterative diagonalization of the Kohn-Sham Hamiltonian. Fermi population of the Kohn-Sham states $\left(k_{B} T=0.2 \mathrm{eV}\right)$ and Pulay mixing of the resulting electronic density have been employed. Energies are extrapolated to $k_{B} T=0 \mathrm{eV}$ and their final evaluation has been performed using the alternative revision of the Perdew, Burke, and Ernzerhof exchange-correlation functional [15]. Simulated STM images have been obtained by applying the Tersoff-Hamann approximation [16] where the tunneling current is proportional to the local density of states. The effect of finite bias has been introduced by adding up contributions from states lying between the Fermi level and the applied voltage. This procedure was already employed successfully to study the $\mathrm{TiO}_{2}$ surface [17]. The voltage considered to model the images is $V_{S}=1 \mathrm{~V}$, similar to the voltage used in the STM experiments (see below). The simulated images have been obtained at a constant density of $3 \times 10^{-6} e / \AA^{3}$, since at this value experimental and theoretical corrugations for the undefected surface are similar.

Figure 2(a) shows an STM image of the clean $\mathrm{TiO}_{2}$ surface recorded at room temperature. For this and all other images in this paper the sample was positively biased at $1.3 \mathrm{~V}$ relative to the tip, implying electron tunneling into unoccupied sample states. In this case the bright rows are assigned to fivefold coordinated $\mathrm{Ti}$ atoms and dark rows to bridging oxygen atoms [17]. Our STM simulations for the clean surface support this interpretation; see Fig. 3(a). In this context, the most important feature of the images is the bright spots (type-A defects) between Ti rows [17].

Exposing the clean samples to a low dose $\left(10^{-2} \mathrm{~L}\right)$ of water at room temperature leads to the situation shown in Fig. 2(b). We again observe the bright Ti rows and type-A defects (protrusions) between the Ti rows. Scrutinizing many similar clean and water exposed STM images reveals two type-A species. Their main difference in appearance is their extent in the [001] direction (vacancy $6.6 \AA$, OH $4.8 \AA$ ) and their apparent height $(0.4 \AA, 0.2 \AA)$. Based on STM simulations, Figs. 3(b) and 3(c), we assign the
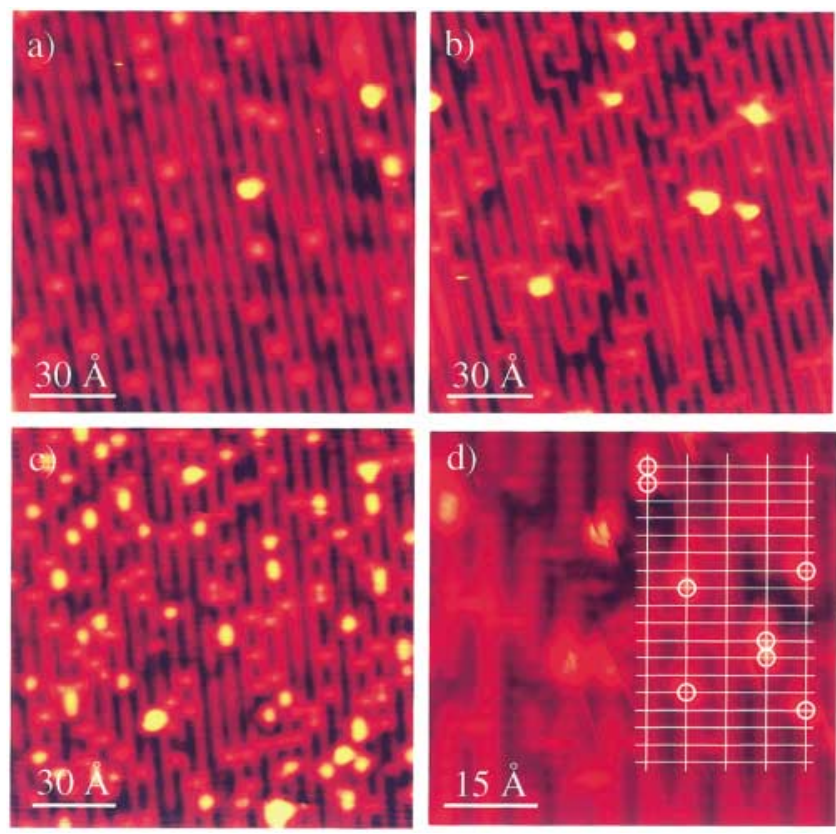

FIG. 2 (color). The $\mathrm{H}_{2} \mathrm{O} / \mathrm{TiO}_{2}(110)$ system at room and low temperature. (a) The bare surface imaged at room temperature. (b) After $10^{-2} \mathrm{~L}$ of water exposure at room temperature. (c) After exposure to $10^{-2} \mathrm{~L}_{\text {of }} \mathrm{H}_{2} \mathrm{O}$ at $180 \mathrm{~K}$, imaged at $160 \mathrm{~K}$. (d) Same conditions as (c). A grid is superimposed to identify the water adsorption site and the location of an $\mathrm{OH}$ group. The small amount of very bright spots in (a) and (b) is unidentified pollution.

broader and slightly higher protrusions [Fig. 2(a)] to be oxygen vacancies and the smaller features [Fig. 2(b)] to be bridging hydroxyl groups. Both vacancies and hydroxyl groups give rise to protrusions but vacancies are clearly more extended than hydroxyl groups due to a stronger delocalization of the two electrons associated with the oxygen vacancy.

The above interpretation is also confirmed by the STM findings. If we compare Figs. 2(a), the clean surface, and 2(b), the surface after water deposition, the number of type-A defects has doubled. For the clean surface in Fig. 2(a) the type-A defects are nearly exclusively $\mathrm{O}$ vacancies, whereas in Fig. 2(b) the type-A defects are $\mathrm{OH}$ groups. If we instead expose the surface to $10^{-2} \mathrm{~L}$ of water at low temperature (180 K), Fig. 2(c), we again observe a doubling of the type-A defect density, but an additional brighter feature is also present, which we ascribe to molecularly adsorbed water. From the atomically resolved STM images [see Fig. 2(d)] we determine the adsorption site of molecular water to be on top fivefold coordinated $\mathrm{Ti}$ atoms. Furthermore, Fig. 2(d) confirms that all $\mathrm{OH}$ groups are situated at bridging oxygen sites; we never observe terminal $\mathrm{OH}$ groups. This suggests that water adsorbs dissociatively in oxygen vacancies and via proton transfer to a neighboring bridging oxygen atom creates two bridging hydroxyl groups per initial vacancy. Oxygen vacancies thus act as the active site for water dissociation on the $\mathrm{TiO}_{2}(110)$ surface. 


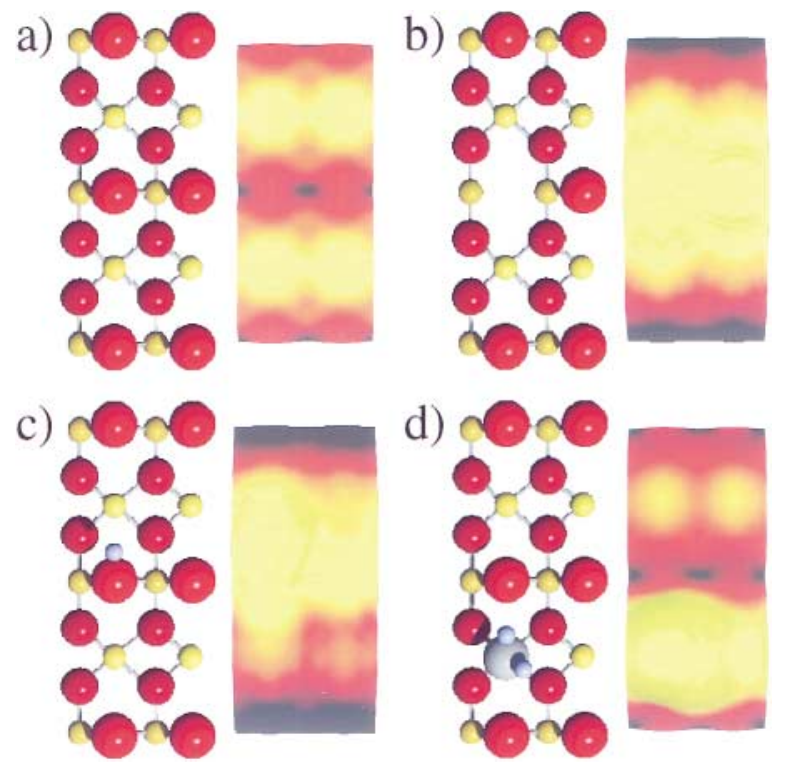

FIG. 3 (color). Ball-and-stick model and corresponding simulated STM image at $1 \mathrm{~V}$ showing the appearance of (a) vacancy-free surface, (b) bridging oxygen vacancy, (c) bridging $\mathrm{OH}$ group, and (d) water molecule on top a fivefold coordinated $\mathrm{Ti}$ atom. Red atoms: $\mathrm{O}$; yellow atoms: Ti; blue atoms: H. In (c) the $\mathrm{OH}$ bond is tilted $\sim 47.9^{\circ}$ with respect to the surface normal. Another equivalent configuration exists with a tilt of $-47.9^{\circ}$, which would make the simulated STM image symmetric.

A prerequisite for dissociation to occur is the mobility of water molecules and thereby their ability to reach the active sites. From time-resolved STM movies [18] we have observed that the water molecules diffuse at $160 \mathrm{~K}$ at time scales of seconds, consistent with Henderson's findings [7].

Temperature programmed desorption (TPD) spectra obtained after submonolayer water deposition at low temperature reveal two peaks at $\sim 290 \mathrm{~K}$ and $\sim 490 \mathrm{~K}$. When annealing higher than $290 \mathrm{~K}$, we no longer observe the bright feature associated with molecularly adsorbed water. Annealing above $490 \mathrm{~K}$ leads to the disappearance of the $\mathrm{OH}$ species, reverting to the initial surface defect density. Correlating TPD and STM data we can thus conclude that the higher temperature peak is associated with recombinative desorption of the hydroxyl species, in agreement with Ref. [19].

DFT calculations support the preceding interpretation in that they show dissociation of water to be energetically possible only at oxygen vacancies (see Fig. 4). When we consider "low" coverages [0.25 ML water coverage, $(2 \times 2)$ super cell], the dissociation of water on the perfect $\mathrm{TiO}_{2}(110)$ surface is endothermic by $0.23 \mathrm{eV}$, in line with [20]. Since the water molecule binds quite strongly to the surface by $0.56 \mathrm{eV}$, the dissociated state is even more endothermic relative to molecular adsorption. The finding of an endothermic dissociation process is in contrast to the result of an earlier calculation by Lindan et al. [9]. Here dissociation is found to be exothermic on the perfect

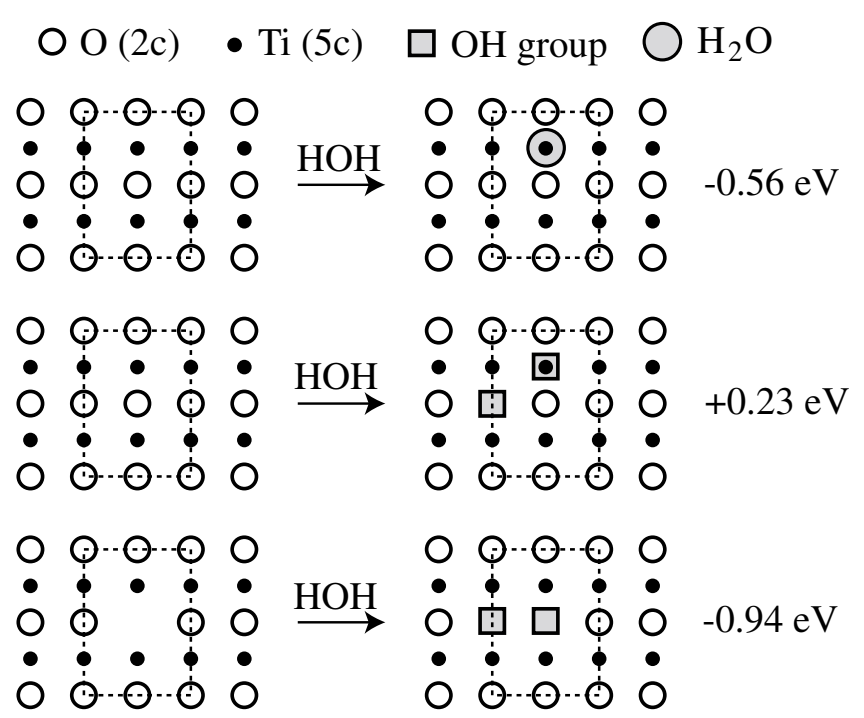

FIG. 4. Energetics for water adsorption processes at low coverages. Top row: molecular adsorption on $\mathrm{TiO}_{2}$; central row: dissociative adsorption; bottom row: dissociative adsorption at an oxygen vacancy. The unit cell employed in the calculations is indicated.

$\mathrm{TiO}_{2}(110)$ surface. We also find this at high coverages. For a $(1 \times 1)$ unit cell dissociation is exothermic relative to molecularly adsorbed water by $0.2 \mathrm{eV}$, the reason being that at this high coverage chains of hydrogen-bonded $\mathrm{OH}$ groups can form along the [001] direction. We note that a small exothermicity may be counteracted by entropic effects and that exothermicity is a necessary, but not sufficient, condition for dissociation. Only for very exothermic processes do we expect the barrier for dissociation to be small. In the calculation by Lindan et al. [9] the unit cell used is such that all $\mathrm{OH}$ groups have nearest neighbors in this direction, and this is likely to be the reason for stronger bonding. The high coverage limit is, however, of limited interest for the understanding of the initial low coverage dissociation of water studied here.

Including now the effect of $0.25 \mathrm{ML}$ of oxygen vacancies, we find dissociation to be strongly exothermic, even with respect to molecularly adsorbed water (see Fig. 4). Dissociation at these vacancies gives rise to two equivalent $\mathrm{OH}$ groups on the surface, both of acidic character, where the water oxygen has been incorporated into the vacancy. The reason for the larger reactivity of the vacancies is clearly associated with the high-energy defect. When water dissociates, the coordination shell of the Ti underneath the vacancy is refilled, resulting in the exothermicity. This electronic effect is very similar to the electronic effect found in the adsorption of various adsorbates on metal surface defects [21].

To further substantiate the results presented above, we carried out a number of quantitative water exposure experiments at room and low temperature for surfaces with different initial surface defect densities (oxygen vacancies and hydroxyl groups originating from the low but finite 

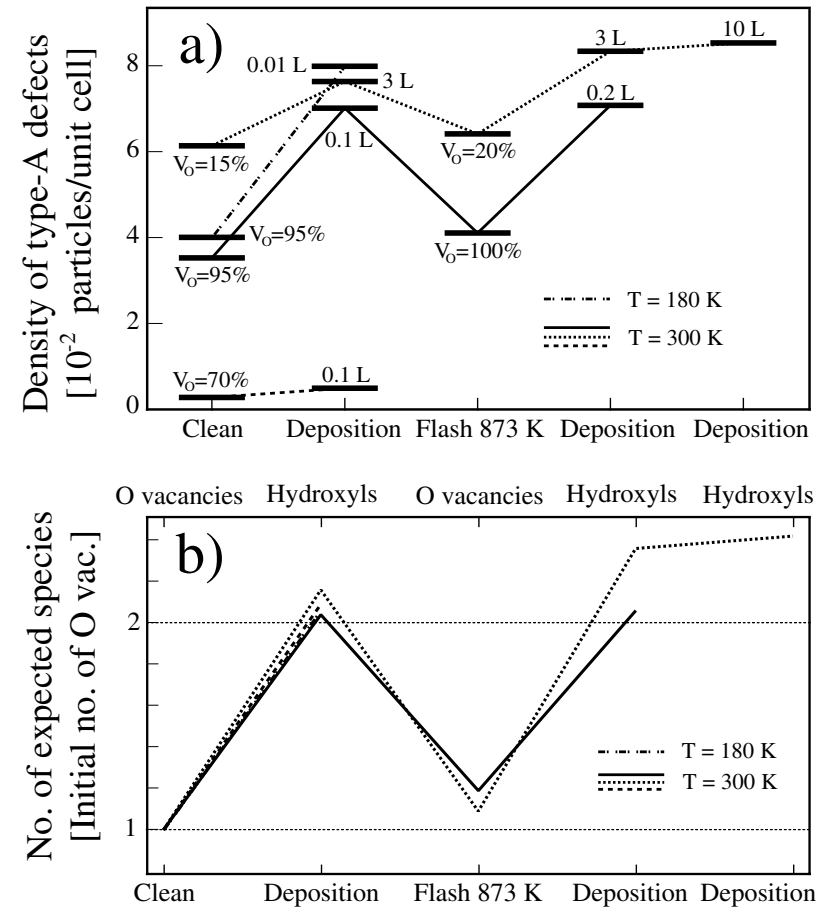

FIG. 5. Quantitative analysis of the number of type-A defects before and after exposure to $\mathrm{H}_{2} \mathrm{O}$. (a) Density of type-A defects for different, widely varying starting configurations. (b) Corrected and normalized type-A defect density plot demonstrating the 1:2 correspondence between the density of oxygen vacancies and hydroxyl groups. Above each step the type of species expected to be present on the surface is noted. In cases where vacancies are the expected species, we correct for the presence of hydroxyl groups by letting two hydroxyl groups count for one vacancy. $V_{\mathrm{O}}$ denotes the percentage of oxygen vacancies in the total density of type-A defects.

water background pressure in the UHV chamber). The defect density can be tuned in a reproducible way by using crystals with varying degrees of bulk reduction and by varying the annealing time in oxygen background and/or UHV. Note that at room temperature, hydroxyl groups have still not desorbed from the surface, as illustrated in Fig. 2(b), while water molecules have desorbed. Figure 5(a) shows a plot of the type-A defect density at different stages in the experiments, where first the clean surface was exposed to a certain amount of water (see "deposition" column). Subsequently, the sample was flash-annealed to $873 \mathrm{~K}$ (indicated with "Flash") and in some cases another deposition was carried out. Upon normalization to the initial vacancy density and correction for the presence of both type-A species it becomes apparent that for every initial vacancy, two hydroxyl groups are created, as shown in Fig. 5(b). Hydroxyl groups are desorbed upon annealing but can be recreated upon another water exposure. This demonstrates unambiguously that the active sites for water dissociation on $\mathrm{TiO}_{2}(110)$ are oxygen vacancies.
In conclusion, from an interplay between experiment and theory we have determined the active site for water dissociation on the rutile $\mathrm{TiO}_{2}(110)$ surface to be an oxygen vacancy. In order to reach this conclusion we have used our ability to distinguish the two defect species, oxygen vacancies and bridging hydroxyl groups, on highresolution STM images.

We acknowledge the financial support from the Danish National Research Foundation through the Center for Atomic-scale Materials Physics (CAMP), from the Danish Research Council through the program "New Design Strategies for Catalysts," and the European Community through the network "OXIDESURFACES." R.S. acknowledges the support from the Swiss National Science Foundation through fellowship 83EU-064104. N.L. acknowledges the support from the European Community HPMF-CT-2000-00431. P. Lindan and U. Diebold are acknowledged for fruitful discussions and G. Thornton for sharing unpublished results.

*Corresponding author.

Email address: fbe@ifa.au.dk.

[1] G. Charlton et al., Phys. Rev. Lett. 78, 495 (1997).

[2] V. E. Henrich and P. A. Cox, The Surface Science of Metal Oxides (Cambridge University Press, Cambridge, 1996).

[3] A. Fujishima and K. Honda, Nature (London) 37, 238 (1972).

[4] A. L. Linsebigler, G. Lu, and J. T. Yates, Jr., Chem. Rev. 95, 735 (1995).

[5] B. Kasemo and J. Gold, Adv. Dent. Res. 13, 8 (1999).

[6] M. B. Hugenschmidt, L. Gamble, and C. T. Campbell, Surf. Sci. 302, 329 (1994).

[7] M. Henderson, Surf. Sci. 355, 151 (1996).

[8] D. Brinkley et al., Surf. Sci. 395, 292 (1998).

[9] P. Lindan, N. Harrison, and M. Gillan, Phys. Rev. Lett. 80, 762 (1998).

[10] U. Diebold et al., Surf. Sci. 411, 137 (1998).

[11] S. Suzuki et al., Phys. Rev. Lett. 84, 2156 (2000).

[12] E. Lægsgaard et al., Rev. Sci. Instrum. 72, 3537 (2001).

[13] D. H. Vanderbilt, Phys. Rev. B 41, 7892 (1990).

[14] J. P. Perdew et al., Phys. Rev. B 46, 6671 (1992).

[15] B. Hammer, L. Hansen, and J. Nørskov, Phys. Rev. B 59, 7413 (1999).

[16] J. Tersoff and D. Hamann, Phys. Rev. Lett. 50, 1998 (1983).

[17] U. Diebold, J. Anderson, K.-O. Ng, and D. Vanderbilt, Phys. Rev. Lett. 77, 1322 (1996).

[18] F. Besenbacher, Rep. Prog. Phys. 59, 1737 (1996). See http://www.ifa.au.dk/camp/hot0021.htm

[19] M. Henderson, Langmuir 12, 5093 (1996).

[20] E. V. Stefanovich and T. N. Truong, Chem. Phys. Lett. 299, 623 (1999).

[21] B. Hammer and J. Nørskov, Adv. Catal. 45, 71 (2000). 